\title{
Spectral phase conjugation via extended phase matching
}

\author{
Mankei Tsang \\ Department of Electrical Engineering, California Institute of Technology, Pasadena, California 91125
}

Received August 12, 2005; revised October 23, 2005; accepted November 17, 2005; posted January 10, 2006 (Doc. ID 63988)

It is shown that the copropagating three-wave-mixing parametric process, with appropriate type-II extended phase matching and pumped with a short second-harmonic pulse, can perform spectral phase conjugation and parametric amplification, which shows a threshold behavior analogous to backward-wave oscillation. The process is also analyzed in the Heisenberg picture, which predicts a spontaneous parametric downconversion rate in agreement with the experimental result reported by Kuzucu et al. [Phys. Rev. Lett. 94, 083601 (2005)]. Applications in optical communications, signal processing, and quantum information processing can be envisaged. (C) 2006 Optical Society of America

OCIS codes: $190.3100,190.4410,190.4970,190.5040,270.4180$.

\section{INTRODUCTION}

In contrast with the more conventional optical phase conjugation schemes that perform phase conjugation with spectral inversion, ${ }^{1}$ spectral phase conjugation (SPC) is the phase conjugation of an optical signal in the frequency domain without spectral inversion. Equivalently, in the time domain, SPC is the phase conjugation and time reversal of the signal complex pulse envelope. ${ }^{2}$ SPC is useful for all-order dispersion and nonlinearity compensation, ${ }^{3,4}$ as well as optical signal processing. ${ }^{5} \mathrm{Al}-$ though SPC has been experimentally demonstrated using photon echo, ${ }^{6,7}$ spectral hole burning, ${ }^{8,9}$ temporal holography, ${ }^{3}$ spectral holography, ${ }^{10}$ and spectral threewave mixing ${ }^{11}$ (TWM), all the demonstrated schemes suffer from the use of cryogenic setups, non-real-time operation, or extremely high pump energy. Pulsed TWM ${ }^{12}$ and four-wave-mixing ${ }^{2,13}$ (FWM) processes in the transversepumping geometry have been theoretically proposed to efficiently perform SPC but have not yet been experimentally realized. All the holographic and wave-mixing schemes also have strict requirements on the transverse beam profile of the signal, limiting their appeal for simultaneous diffraction and dispersion compensation applications.

There is a correspondence between classical SPC and quantum coincident frequency entanglement, as shown in Ref. 14 for the transversely pumped $\mathrm{TWM}^{12,15}$ and $\mathrm{FWM}^{2,13}$ processes. It is then interesting to see if other coincident frequency entanglement schemes are also capable of performing SPC when an input signal is present. This paper studies one of such schemes, which makes use of extended phase matching ${ }^{16}$ (EPM) and has been experimentally demonstrated ${ }^{17}$ in a periodically poled potassium titanyl phosphate (PPKTP) crystal. $^{18}$ It is shown in Section 3, for the first time to the author's knowledge, that this EPM scheme is indeed capable of performing SPC and optical parametric amplification (OPA) more efficiently than previous proposals.

The analysis also yields a surprising result, namely, that the parametric gain can be theoretically infinite even for a pump pulse with finite energy, analogous to backward-wave oscillation, where counterpropagating waves are parametrically coupled and can give rise to mirrorless optical parametric oscillation ${ }^{19-25}$ (OPO). The reason for the similarity is that, in the scheme presented here, even though the signal and the idler copropagate with the pump pulse in the laboratory frame, they counterpropagate in the frame of the moving pump pulse because one is faster than the pump and one is slower. Hence the moving pump pulse provides both an effective cavity and a parametric gain, leading to oscillation. In reality, however, the interaction among the pulses should be ultimately limited by the finite device length. It is shown in Section 4, with a Laplace analysis, that the parametric gain should abruptly increase above the threshold, where infinite gain is predicted by the Fourier analysis, but a finite medium length would always limit the gain to a finite value. Still, as previous proposals of TWM mirrorless OPO have never been experimentally achieved owing to the requirement of a continuous-wave $(\mathrm{CW})$ pump and the difficulty in phase matching counterpropagating waves, the presented analysis suggests the exciting possibility that mirrorless OPO can be realized with an ultrashort pump pulse and a practical poling period for phase matching of copropagating modes, if a long-enough medium can be fabricated and parasitic effects can be controlled. By analysis of the scheme in the Heisenberg picture in Section 5, a high spontaneous parametric downconversion rate is also predicted, in excellent agreement with the experimental result reported in Ref. 17 . The result should be useful for many quantum information processing applications, such as quantum-enhanced synchronization ${ }^{26}$ and multiphoton entanglement for quantum cryptography. ${ }^{27}$ Finally, numerical results are presented in Section 6, which confirm the theoretical predictions.

\section{SETUP}

Consider the copropagating TWM process (Fig. 1), assuming that the basic type-II phase-matching condition $\left(k_{s}\right.$ 


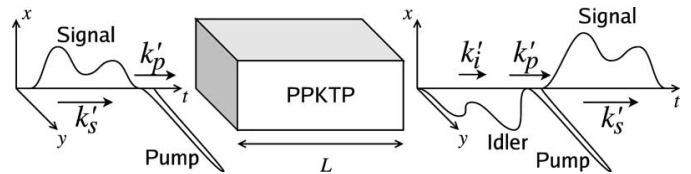

Fig. 1. Schematic of SPC via type-II EPM. The signal and idler pulses, in orthogonal polarizations, have carrier frequencies of $\omega_{s}$ and $\omega_{i}$, while the pump pulse has a carrier frequency of $\omega_{p}=\omega_{s}$ $+\omega_{i}$. The EPM condition requires that the signal and the idler counterpropagate with respect to the pump, which should be much shorter than the input signal.

$+k_{i}=k_{p}+2 \pi / \Lambda$ ), with a quasi-phase-matching period $\Lambda$, is satisfied. The coupled-mode equations are

$$
\begin{aligned}
& \frac{\partial A_{p}}{\partial z}+k_{p}^{\prime} \frac{\partial A_{p}}{\partial t}=j \chi_{p} A_{s} A_{i}, \\
& \frac{\partial A_{s}}{\partial z}+k_{s}^{\prime} \frac{\partial A_{s}}{\partial t}=j \chi_{s} A_{p} A_{i}^{*}, \\
& \frac{\partial A_{i}^{*}}{\partial z}+k_{i}^{\prime} \frac{\partial A_{i}^{*}}{\partial t}=-j \chi_{i} A_{p}^{*} A_{s},
\end{aligned}
$$

where $A_{p}$ is the pump pulse envelope of carrier frequency $\omega_{p}, A_{s, i}$ are the signal and idler envelopes of frequencies $\omega_{s}$ and $\omega_{i}$, respectively, $k_{p, s, i}^{\prime}$ are the group delays of the three modes, $\chi_{p, s, i} \equiv \omega_{p, s, i} \chi^{(2)} /\left(2 c n_{p, s, i}\right)$ are the nonlinear coupling coefficients, $\omega_{p, s, i}$ are the center frequencies of the modes such that $\omega_{s}+\omega_{i}=\omega_{p}$, and $n_{p, s, i}$ are the refractive indices. Group-velocity dispersion within each mode and diffraction are neglected. Define $\tau \equiv t-k_{p}^{\prime} z$ as the retarded time coordinate that follows the propagating pump pulse. The change of coordinates yields

$$
\begin{array}{r}
\frac{\partial A_{p}}{\partial z}=j \chi_{p} A_{s} A_{i}, \\
\frac{\partial A_{s}}{\partial z}+\left(k_{s}^{\prime}-k_{p}^{\prime}\right) \frac{\partial A_{s}}{\partial \tau}=j \chi_{s} A_{p} A_{i}^{*}, \\
\frac{\partial A_{i}^{*}}{\partial z}+\left(k_{i}^{\prime}-k_{p}^{\prime}\right) \frac{\partial A_{i}^{*}}{\partial \tau}=-j \chi_{i} A_{p}^{*} A_{s} .
\end{array}
$$

Throughout the theoretical analysis, the pump is assumed to be undepleted and unchirped, so that $A_{p}$ $=A_{p 0}\left(t-k_{p}^{\prime} z\right)=A_{p 0}(\tau)$, hereafter regarded as real without loss of generality.

\section{FOURIER ANALYSIS}

Equations (5) and (6) are space invariant if the nonlinear medium length $L$ is much longer than the signal or idler spatial pulse width in the frame of $z$ and $\tau$, or

$$
L \gg \frac{T_{s, i}}{\left|k_{s, i}^{\prime}-k_{p}^{\prime}\right|},
$$

where $T_{s, i}$ is the signal or idler pulse width. One can then perform Fourier transform on the equations with respect to $z$, as defined by the following:

$$
\begin{aligned}
& \widetilde{A}_{s}(\kappa, \tau) \equiv \int_{-\infty}^{\infty} A_{s}(z, \tau) \exp (-j \kappa z) \mathrm{d} z, \\
& \tilde{A}_{i}^{*}(\kappa, \tau) \equiv \int_{-\infty}^{\infty} A_{i}^{*}(z, \tau) \exp (-j \kappa z) \mathrm{d} z .
\end{aligned}
$$

Notice that $\widetilde{A}_{i}^{*}$ is defined as the Fourier transform after the conjugation of $A_{i}$. The coupled-mode equations become

$$
\begin{aligned}
& j \kappa \widetilde{A}_{s}+\left(k_{s}^{\prime}-k_{p}^{\prime}\right) \frac{\partial \widetilde{A}_{s}}{\partial \tau}=j \chi_{s} A_{p 0}(\tau) \tilde{A}_{i}^{*}, \\
& j \kappa \widetilde{A}_{i}^{*}+\left(k_{i}^{\prime}-k_{p}^{\prime}\right) \frac{\partial \widetilde{A}_{i}^{*}}{\partial \tau}=-j \chi_{i} A_{p 0}(\tau) \tilde{A}_{s} .
\end{aligned}
$$

Let

$$
\gamma_{s} \equiv k_{s}^{\prime}-k_{p}^{\prime}, \quad \gamma_{i} \equiv k_{i}^{\prime}-k_{p}^{\prime}, \quad r \equiv\left|\frac{\gamma_{s} \chi_{i}}{\gamma_{i} \chi_{s}}\right| .
$$

Consider the case in which $\gamma_{s}$ and $\gamma_{i}$ are nonzero and have opposite signs, implying that the signal and the idler propagate in opposite directions with respect to the pump. This can be achieved for a range of wavelengths in KTP. Without loss of generality, assume that $\gamma_{s}>0$ and $\gamma_{i}<0$, so that $k_{s}^{\prime}>k_{p}^{\prime}>k_{i}^{\prime}$. Making the following substitutions,

$$
A=\sqrt{r} \tilde{A}_{s} \exp \left(j \frac{\kappa}{\gamma_{s}} \tau\right), \quad B=\tilde{A}_{i}^{*} \exp \left(j \frac{\kappa}{\gamma_{i}} \tau\right),
$$

one obtains

$$
\begin{aligned}
& \frac{\partial A}{\partial \tau}=j \sqrt{\left|\frac{\chi_{s} \chi_{i}}{\gamma_{s} \gamma_{i}}\right|} A_{p 0}(\tau) B \exp \left[j \kappa\left(\frac{1}{\gamma_{s}}-\frac{1}{\gamma_{i}}\right) \tau\right], \\
& \frac{\partial B}{\partial \tau}=j \sqrt{\left|\frac{\chi_{s} \chi_{i}}{\gamma_{s} \gamma_{i}}\right|} A_{p 0}(\tau) A \exp \left[-j \kappa\left(\frac{1}{\gamma_{s}}-\frac{1}{\gamma_{i}}\right) \tau\right] .
\end{aligned}
$$

Owing to linear space invariance, the wave-mixing process cannot generate new spatial frequencies $(\kappa)$ for $A$ and $B$. The magnitude of $\kappa$ then depends only on the initial bandwidths of $A$ and $B$ and is of the order of $2 \pi \gamma_{s, i} / T_{s, i}$. As a result, if the pump pulse width $T_{p}$ is much shorter than the minimum period of the detuning factor $\exp \left[ \pm j \kappa\left(1 / \gamma_{s}\right.\right.$ $\left.\left.-1 / \gamma_{i}\right) \tau\right]$, or

$$
T_{p} \ll\left|\frac{2 \pi}{\kappa\left(1 / \gamma_{s}-1 / \gamma_{i}\right)}\right| \sim\left|\frac{T_{s, i}}{\gamma_{s, i}\left(1 / \gamma_{s}-1 / \gamma_{i}\right)}\right|,
$$

the pump can effectively sample the detuning factor, say, at $\tau=0$. Defining a normalized coupling function,

$$
g(\tau) \equiv \sqrt{\left|\frac{\chi_{s} \chi_{i}}{\gamma_{s} \gamma_{i}}\right|} A_{p 0}(\tau),
$$

one can obtain two simple coupled-mode equations: 


$$
\begin{aligned}
& \frac{\partial A}{\partial \tau}=j g(\tau) B, \\
& \frac{\partial B}{\partial \tau}=j g(\tau) A .
\end{aligned}
$$

Because the signal and the idler counterpropagate with respect to the pump, the signal should begin to mix with the pump at the leading edge of the pump pulse, say, at $\tau=-T_{p} / 2$, while the idler should begin to mix at the trailing edge of the pump, say, at $\tau=T_{p} / 2$. The solutions of Eqs. (18) and (19) can then be written as

$$
\begin{aligned}
A(\kappa, \tau)= & \sec (G)\left\{A\left(\kappa,-\frac{T_{p}}{2}\right) \cos \left[\int_{T_{p} / 2}^{\tau} g\left(\tau^{\prime}\right) \mathrm{d} \tau^{\prime}\right]\right. \\
& \left.+j B\left(\kappa, \frac{T_{p}}{2}\right) \sin \left[\int_{-T_{p} / 2}^{\tau} g\left(\tau^{\prime}\right) \mathrm{d} \tau^{\prime}\right]\right\}, \\
B(\kappa, \tau)= & \sec (G)\left\{j A\left(\kappa,-\frac{T_{p}}{2}\right) \sin \left[\int_{T_{p} / 2}^{\tau} g\left(\tau^{\prime}\right) \mathrm{d} \tau^{\prime}\right]\right. \\
& \left.+B\left(\kappa, \frac{T_{p}}{2}\right) \cos \left[\int_{-T_{p} / 2}^{\tau} g\left(\tau^{\prime}\right) \mathrm{d} \tau^{\prime}\right]\right\},
\end{aligned}
$$

where

$$
G \equiv \int_{-T_{p} / 2}^{T_{p} / 2} g(\tau) \mathrm{d} \tau \approx \int_{-\infty}^{\infty} g(\tau) \mathrm{d} \tau .
$$

The input signal pulse is required to be placed in advance of the pump (by $t_{s} \gg T_{s}$ ), and the input idler pulse is required to be placed behind the pump (delayed by $t_{i} \gg T_{i}$ ), so that the signal and the idler overlap the pump pulse only inside the nonlinear medium. Consequently, the output solutions are

$$
\begin{aligned}
A_{s}(L, t)= & A_{s 0}\left(t-k_{s}^{\prime} L+t_{s}\right) \sec (G) \\
& +j \frac{1}{\sqrt{r}} A_{i 0}^{*}\left[-\frac{1}{r}\left(t-k_{s}^{\prime} L-t_{i}\right)\right] \tan (G), \\
A_{i}(L, t)= & A_{i 0}\left(t-k_{i}^{\prime} L+t_{i}\right) \sec (G) \\
& +j \sqrt{r} A_{s 0}^{*}\left[-r\left(t-k_{i}^{\prime} L+t_{s}\right)\right] \tan (G) .
\end{aligned}
$$

To see how the device is able to perform SPC, assume that the center frequencies of the two modes are the same, $\omega_{s}=\omega_{i}, \chi_{s}=\chi_{i}$, and the type-II EPM condition,

$$
k_{s}^{\prime}+k_{i}^{\prime}=2 k_{p}^{\prime}, \quad k_{s}^{\prime} \neq k_{i}^{\prime},
$$

which depends on the material dispersion properties and typically occurs at a single set of center frequencies, is satisfied. ${ }^{16}$ Then $r=1$, and the output idler becomes the phase-conjugated and time-reversed replica of the input signal, if the input idler is zero. SPC is hence performed. The SPC efficiency $\eta$, or the idler gain, defined as the output idler fluence divided by the input signal fluence, is

$$
\eta \equiv \frac{\int_{-\infty}^{\infty}\left|A_{i}(L, t)\right|^{2} \mathrm{~d} t}{\int_{-\infty}^{\infty}\left|A_{s}(0, t)\right|^{2} \mathrm{~d} t}=\tan ^{2}(G) .
$$

This SPC efficiency can be fundamentally higher than that of the transversely pumped TWM device ${ }^{12}$ because of two reasons. One is the copropagation of the three pulses, which makes $G$ higher than a similar parameter in the latter case by a factor of $\left(1-k_{p}^{\prime} / k_{s}^{\prime}\right)^{-1}$, of the order of 40 for KTP. The second reason is that for $\eta>1$, owing to the tangent function dependence, the SPC efficiency of the EPM scheme increases with respect to $G$ much faster than that of the latter, which only depends on a similar parameter exponentially. That said, the transversely pumped FWM device $^{13}$ can still be more efficient in the small-gain regime $\eta<1$ if a highly nonlinear material, such as polydiacetylene, is used. Furthermore, the EPM device requires a longer nonlinear medium length by a factor of (1 $\left.-k_{p}^{\prime} / k_{s}^{\prime}\right)^{-1}$ and depends crucially on the material dispersion, thus severely limiting the flexibility in the choice of operating wavelengths.

Equations (23) and (24) are obtained from the analysis of the coupled-mode equations (5) and (6), after Fourier transform with respect to $z$ is performed. The solutions are therefore formally valid only when the nonlinear medium length $L$ goes to infinity. In practice, in the moderate-gain regime $\eta \sim O(1)$, the approximation given by expression (7) should be adequate, where the length $L$ can be, say, ten times larger than the signal spatial pulse width in the frame of $z$ and $\tau$. Numerical analysis in Section 6 will validate the accuracy of the Fourier solutions.

\section{LAPLACE ANALYSIS}

Intriguingly, the Fourier solutions, Eqs. (23) and (24), have the same form as those of backward-wave oscillation, ${ }^{19-25}$ suggesting that the device studied here, with an ultrashort pump pulse and a practical quasiphase-matching period ( $\Lambda=46 \mu \mathrm{m}$ as reported in Ref. 18), can also perform mirrorless OPO, as long as $k_{s, i}^{\prime}>k_{p}^{\prime}$ $>k_{i, s}^{\prime}$. However, the prediction of infinite gain is based on the assumption of infinite medium length and therefore may not be valid. In this case, Laplace transform should be used.

For the CW-pumped mirrorless OPO schemes, a Laplace analysis ${ }^{28}$ with respect to time shows that, beyond threshold, poles appear on the right-hand plane in the Laplace domain, meaning that the temporal impulse response increases exponentially with time, leading to self-oscillation when enough time has elapsed. The same procedures of utilizing the two-sided Laplace transform ${ }^{29}$ as in Ref. 28 are followed here in order to be consistent with the relevant literature, but, since the proposed scheme is the opposite limit of the CW devices, the Laplace transform should be performed with respect to $z$ instead:

$$
\bar{A}_{s}(p, \tau) \equiv \int_{-\infty}^{\infty} A_{s}(z, \tau) \exp (-p z) \mathrm{d} z
$$




$$
\bar{A}_{i}^{*}(p, \tau) \equiv \int_{-\infty}^{\infty} A_{i}^{*}(z, \tau) \exp (-p z) \mathrm{d} z .
$$

For simplicity but without affecting the qualitative behavior of the solutions, it is assumed that the pump pulse is square, there is no input idler, $\gamma=\gamma_{s}=-\gamma_{i}$, and $\chi=\chi_{s}$ $=\chi_{i}$. The output solutions in the Laplace domain are then given by

$$
\begin{gathered}
\bar{A}_{s}\left(p, \frac{T_{p}}{2}\right)=\frac{\sqrt{1-P^{2}} \csc \left(G \sqrt{1-P^{2}}\right)}{P+\sqrt{1-P^{2}} \cot \left(G \sqrt{1-P^{2}}\right)} \bar{A}_{s}\left(p,-\frac{T_{p}}{2}\right), \\
\bar{A}_{i}^{*}\left(p,-\frac{T_{p}}{2}\right)=\frac{-j}{P+\sqrt{1-P^{2}} \cot \left(G \sqrt{1-P^{2}}\right)} \bar{A}_{s}\left(p,-\frac{T_{p}}{2}\right), \\
P \equiv \frac{p}{\chi A_{p 0}}, \quad G \equiv \chi A_{p 0}\left(\frac{T_{p}}{\gamma}\right) .
\end{gathered}
$$

If we let $p=j \kappa$, the transfer functions in Eqs. (29) and (30) are well known to be low-pass filters, ${ }^{30}$ the bandwidth of which decreases as $G$ increases. If the spatial bandwidth of the input signal, of the order of $\gamma / T_{s}$, is much smaller than the bandwidth of the low-pass filters, the transfer functions can be regarded as flat-top functions, and, by plugging $P=0$ into Eqs. (29) and (30), one can recover the Fourier solutions in Eqs. (23) and (24). For $G \ll 1$, the transfer functions are sinc functions with a bandwidth $\sim \gamma / T_{p}$, so the Fourier solutions are valid if $T_{p} \ll T_{s}$, which is essentially the same assumption used in the Fourier analysis, expression (16). As $G$ increases and the filter bandwidth decreases, however, the Fourier solutions become less and less accurate for a finite-bandwidth input signal.

The poles of the transfer functions, $p_{\infty}$, can be obtained by setting the denominators of Eqs. (29) and (30) to zero,

$$
p_{\infty}+\sqrt{\left(\chi A_{p 0}\right)^{2}-p_{\infty}^{2}} \cot \left[G \sqrt{1-p_{\infty}^{2} /\left(\chi A_{p 0}\right)^{2}}\right]=0 .
$$

Figure 2 plots the normalized poles $p_{\infty} /\left(\chi A_{p 0}\right)$ against

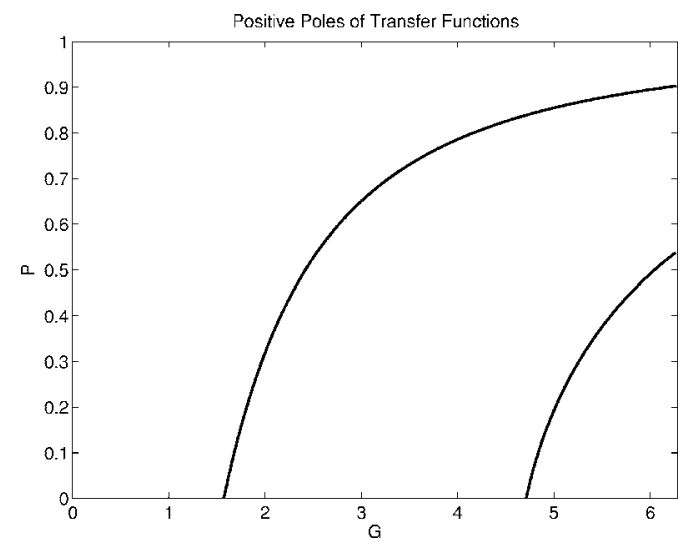

Fig. 2. Normalized poles $p_{\infty} /\left(\chi A_{p 0}\right)$ plotted against $G$, obtained by numerically solving Eq. (32), indicating the onset of spatial instability beyond the threshold $G>\pi / 2$. More poles appear as $G$ is increased.
$G$. Positive poles begin to appear when $G>\pi / 2$; hence the spatial impulse response increases exponentially with respect to $z$ beyond threshold.

It is interesting to compare the scheme studied here with the case in which the pump, signal, and idler have degenerate group delays $\left(k_{p}^{\prime}=k_{s}^{\prime}=k_{i}^{\prime}\right) .{ }^{31}$ The coupled-mode equations of the latter case are

$$
\frac{\partial A_{s, i}(z, \tau)}{\partial z}=j \chi A_{p 0}(\tau) A_{i, s}^{*}(z, \tau),
$$

where the $\tau$ derivatives vanish. The solutions are easily seen to be

$A_{s, i}(z, \tau)=A_{s, i}(0, \tau) \cosh \left[\chi A_{p 0}(\tau) z\right]+j A_{i, s}^{*}(0, \tau) \sinh \left[\chi A_{p 0}(\tau) z\right]$.

This corresponds to the $G \rightarrow \infty$ limit of the former scheme, where $p_{\infty} /\left(\chi A_{p 0}\right) \rightarrow 1$ and all the poles approach the growth rate of the degenerate case, $\chi A_{p 0}$.

\section{SPONTANEOUS PARAMETRIC DOWN CONVERSION}

Given the input-output signal-idler relationship in Eqs. (23) and (24), it is straightforward to obtain a quantum picture of the parametric process in the moderate-gain a regime by replacing the signal and idler envelopes with Heisenberg operators, so that

$$
\begin{aligned}
& \hat{A}_{s}=\hat{A}_{s 0} \sec (G)+j \hat{A}_{i 0}^{\dagger} \tan (G), \\
& \hat{A}_{i}=j \hat{A}_{s 0}^{\dagger} \tan (G)+\hat{A}_{i 0} \sec (G) .
\end{aligned}
$$

If the inputs are Fock states,

$$
\begin{gathered}
n_{s, i} \equiv\left\langle\hat{A}_{s, i}^{\dagger} \hat{A}_{s, i}\right\rangle=\left\langle\hat{A}_{s, i} \hat{A}_{s, i}^{\dagger}\right\rangle-1, \\
\left\langle\hat{A}_{s 0}^{\dagger} \hat{A}_{i 0}\right\rangle=\left\langle\hat{A}_{i 0}^{\dagger} \hat{A}_{s 0}\right\rangle=\left\langle\hat{A}_{s 0} \hat{A}_{i 0}^{\dagger}\right\rangle=\left\langle\hat{A}_{i 0} \hat{A}_{s 0}^{\dagger}\right\rangle=0 .
\end{gathered}
$$

The average output photon number of each mode is

$$
\begin{aligned}
& n_{s}=n_{s 0} \sec ^{2}(G)+\left(n_{i 0}+1\right) \tan ^{2}(G), \\
& n_{i}=n_{i 0} \sec ^{2}(G)+\left(n_{s 0}+1\right) \tan ^{2}(G) .
\end{aligned}
$$

The average number of spontaneously generated photon pairs per pump pulse is therefore the same as the idler gain, or $\eta=\tan ^{2}(G)$. Moreover, the unitary transform given by Eqs. (35) and (36) has the same form as the CW FWM process. One then expects the photon wave function to be similarly given by ${ }^{32}$

$$
|\psi\rangle=\cos (G) \sum_{n=0}^{\infty} \sin ^{n}(G)|n\rangle_{s}|n\rangle_{i},
$$

where $|n\rangle_{s, i}$ is the Fock state in the signal or idler mode. The scheme thus has a significant advantage in efficiency and robustness for multiphoton entanglement, compared with other schemes that often require feedback. ${ }^{33}$ The efficient multiphoton coincident frequency entanglement should be useful for quantum-enhanced synchronization ${ }^{26}$ and quantum cryptography applications. ${ }^{27}$ 
The preceding quantum analysis assumes that there is only one spatial mode in each signal or idler mode and is accurate only when the Fourier solutions are accurate. This restricts the applicability of the quantum analysis to the moderate-gain regime $\eta \sim O(1)$, depending on how closely the assumption in expression (7) is observed. It is beyond the scope of this paper to investigate what happens in the quantum picture when more than one spatial mode is involved, but, qualitatively, one expects that each spatial mode should have a varying parametric gain depending on the spatial frequency, as suggested by the Laplace solutions in Eqs. (29) and (30), so the photon wave function would be given by a superposition of simultaneous eigenstates of spatial frequency and photon number.

Using the parameters described in Refs. 17 and 18, where $\quad \lambda_{0}=1584 \mathrm{~nm}, \quad \chi^{(2)}=7.3 \mathrm{pm} / \mathrm{V}, \quad n_{0}=2, \quad \gamma=1.5$ $\times 10^{-10} \mathrm{~s} / \mathrm{m}, T_{p}=100 \mathrm{fs}$, average pump power $=350 \mathrm{~mW}$, diameter $=200 \mu \mathrm{m}$, and pump repetition rate $f_{\text {rep }}$ $=80 \mathrm{MHz}$, the spontaneously generated photon pairs per second is theoretically given by $f_{\text {rep }} \tan ^{2}(G) \approx f_{\text {rep }} G^{2}=3.6$ $\times 10^{6} / \mathrm{s}$, in excellent agreement with the experimental result reported in Ref. 17, which is $\sim 4 \times 10^{6} / \mathrm{s}$. $G$ is then given by $\sim 0.2$, so the operations of SPC, OPA, and multiphoton entanglement $(G>\pi / 4)$ should be realizable by increasing the pump field amplitude.

\section{NUMERICAL ANALYSIS}

Equations (5) and (6) are solved numerically via a Fourier split-step approach to confirm the above theoretical predictions. Figure 3 plots the intensities and phases of input signal, output signal, and output idler from the numerical analysis when $G=\pi / 4$. The plots clearly show that the output idler is the time-reversed and phase-conjugated replica of the signal.

Figure 4 plots the numerical signal gain and idler gain compared with Fourier theory for $0<G \leqslant \pi / 3$. The numerical results are all within $3 \%$ of the theoretical values.

Figure 5 plots the idler gain on the logarithmic scale for a wider range of $G$ 's and two different lengths, obtained from the numerical analysis of the complete TWM equations (4)-(6), with a single photon as the input signal, approximately emulating parametric fluorescence. For the $L=10 \mathrm{~cm}$ case, the curve can be clearly separated into three regimes. For $G<\pi / 2$ and moderate gain $(\eta \sim 0 \mathrm{~d} B)$, the idler gain approximately follows the Fourier solution (dashed curve). For $G>\pi / 2$, the system becomes unstable and an exponential growth (linear ramp on the logarithmic curve) is observed, until the pump is significantly depleted, parametric oscillation occurs, and the exponential growth abruptly stops.

For $L=1 \mathrm{~cm}$, the numerical solution departs from theory for a smaller $G$, and the slope of the logarithmic curve in the unstable regime, proportional to $L$, is too small to initiate oscillation in the parameter range of interest.

A medium length of $10 \mathrm{~cm}$ may be pushing the limit of current technology. Even if one is able to fabricate such a long periodically poled nonlinear crystal, the effectivemedium length is always limited by parasitic effects, such as diffraction, group-velocity dispersion, and competing
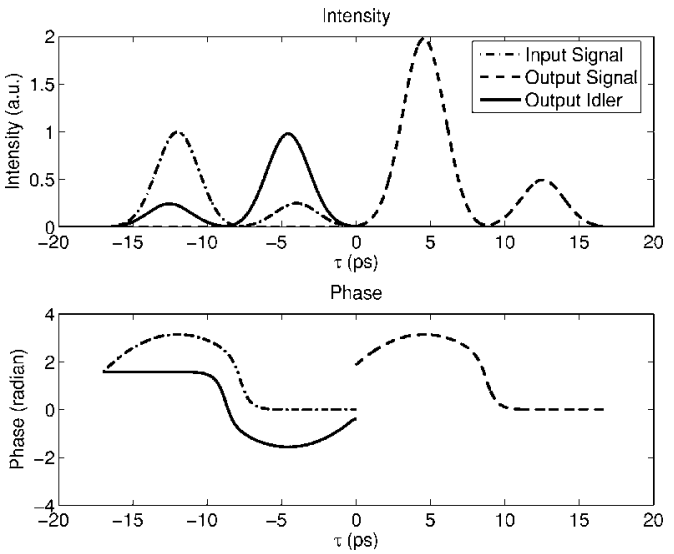

Fig. 3. Plots of intensity and phase of the input signal, output signal, and output idler, from numerical analysis of Eqs. (5) and (6). Parameters used are $k_{p}^{\prime}=1 /\left(1.5 \times 10^{8} \mathrm{~ms}^{-1}\right), k_{s}^{\prime}=1.025 k_{p}^{\prime}, k_{i}$ $=0.975 k_{p}^{\prime}, \quad T_{p}=100 \mathrm{fs}, \quad T_{s}=2 \mathrm{ps}, \quad L=10 \mathrm{~cm}, \quad t_{s}=4 T_{s}, \quad$ beam diameter $=200 \mu \mathrm{m}, A_{s 0}=0.5 \exp \left[-\left(t-2 T_{s}\right)^{2} /\left(2 T_{s}^{2}\right)\right]-\exp [-(1+0.5 j)$ $\left.\times\left(t+2 T_{s}\right)^{2} /\left(2 T_{s}^{2}\right)\right], A_{p 0}=\exp \left[-t^{2} /\left(2 T_{p}^{2}\right)\right]$, and $G=\pi / 4$. The plots clearly show that the idler is the time-reversed and phaseconjugated replica, i.e., SPC, of the signal.

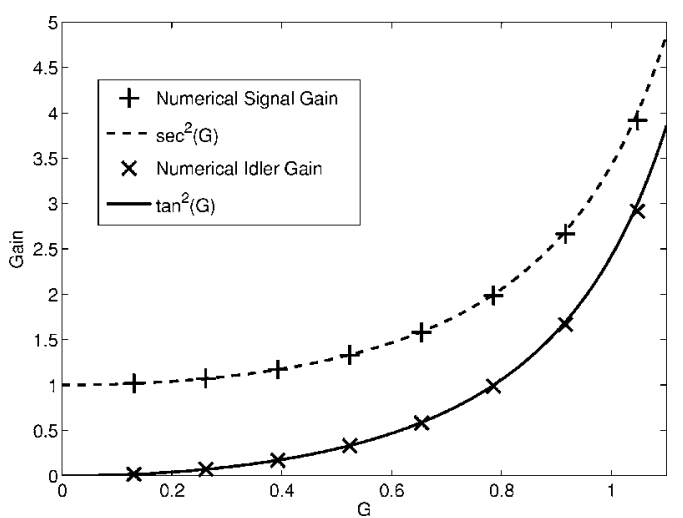

Fig. 4. Signal gain $\eta+1$ and idler gain $\eta$ versus $G$ from numerical analysis compared with theory. See the caption of Fig. 3 for parameters used.

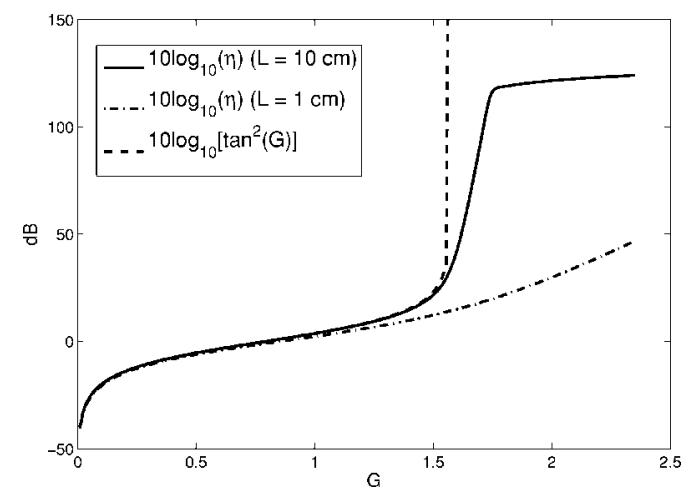

Fig. 5. Plot of numerical idler gain $\eta$ in decibels against $G$ for $L=10 \mathrm{~cm}$ (solid curve) and $L=1 \mathrm{~cm}$ (dashed-dotted curve), compared with the Fourier theory (dashed curve), $\tan ^{2}(G)$ in decibels. Three distinct regimes can be observed for the $L=10 \mathrm{~cm}$ case: the moderate-gain regime where the Fourier theory is accurate, the unstable regime where the gain increases exponentially, and the oscillation regime where significant pump depletion occurs. For $L=1 \mathrm{~cm}$, the medium is not long enough for oscillation to occur in the parameter range of interest. 
third-order nonlinearities, so it might be difficult to fabricate an ideal EPM device for the aforementioned purposes. For instance, in the experiment by Kuzucu et al., ${ }^{17}$ the diameter of the beam is $W \sim 200 \mu \mathrm{m}$, so the characteristic diffraction length is $\sim W^{2} / \lambda_{0}=4 \mathrm{~cm}$, and the characteristic group-velocity dispersion length is $20 \mathrm{~cm}$ according to Ref. 16, all of which are of the order of the medium length required for mirrorless OPO. That said, techniques such as diffusion bonding ${ }^{34}$ can be used to increase the length of a nonlinear crystal, diffraction can be eliminated by waveguiding, and there exists a variety of methods to compensate for group-velocity dispersion and thirdorder nonlinearities. ${ }^{35}$ Hence with careful engineering, fabricating an EPM device for the proposed applications is still a distinct possibility.

\section{CONCLUSION}

In summary, it is proven that the copropagating threewave-mixing process, with appropriate extended phase matching and pumped with a short second-harmonic pulse, is capable of performing spectral phase conjugation, parametric amplification, and efficient multiphoton entanglement. The main technical challenges of experimental implementation seem to be the long medium length required and the control of parasitic effects such as diffraction, group-velocity dispersion, and competing third-order nonlinearities. However, a shorter proof-ofconcept device has already been experimentally realized for the purposes of broadband second-harmonic generation $^{18}$ and coincident frequency entanglement, ${ }^{17}$ so it is not unrealistic to expect that a longer device can be fabricated for the proposed applications, which should be useful for optical communications, signal processing, and quantum information processing.

Theoretically, much remains to be explored. The study of parasitic effects, not considered in this paper, is vital for experimental realization. The analysis of the ultrashort-pump limit can be potentially generalized to other TWM and FWM geometries, and the quantum analysis of this limit is by no means complete. In conclusion, the analysis presented here should stimulate further experimental and theoretical investigations of a new class of parametric devices.

The author thanks Demetri Psaltis for helpful discussions and a reviewer for pointing out Refs. 19-22.

The author may be reached by e-mail at mankei @sunoptics.caltech.edu.

\section{REFERENCES}

1. A. Yariv, D. Fekete, and D. M. Pepper, "Compensation for channel dispersion by nonlinear optical phase conjugation," Opt. Lett. 4, 52-54 (1979).

2. D. A. B. Miller, "Time reversal of optical pulses by fourwave mixing," Opt. Lett. 5, 300-302 (1980).

3. C. Joubert, M. L. Roblin, and R. Grousson, "Temporal reversal of picosecond optical pulses by holographic phase conjugation," Appl. Opt. 28, 4604-4612 (1989).

4. M. Tsang and D. Psaltis, "Dispersion and nonlinearity compensation by spectral phase conjugation," Opt. Lett. 28, 1558-1560 (2003).

5. D. M. Marom, D. Panasenko, P.-C. Sun, Y. T. Mazurenko, and Y. Fainman, "Real-time spatial-temporal signal processing with optical nonlinearities," IEEE J. Sel. Top. Quantum Electron. 7, 683-693 (2001).

6. N. W. Carlson, L. J. Rothberg, A. G. Yodh, W. R. Babbitt, and T. W. Mossberg, "Storage and time reversal of light pulses using photon echoes," Opt. Lett. 8, 483-485 (1983).

7. V. L. da Silva, Y. Silberberg, J. P. Heritage, E. W. Chase, M. A. Saifi, and M. J. Andrejco, "Femtosecond accumulated photon echo in Er-doped fibers," Opt. Lett. 16, 1340-1342 (1991).

8. A. Rebane, J. Aaviksoo, and J. Kuhl, "Storage and time reversal of femtosecond light signals via persistent spectral hole burning holography," Appl. Phys. Lett. 54, 93-95 (1989).

9. S. Fraigne, J. P. Galaup, J. L. Le Gouet, B. Bousquet, L. Canioni, M. Joffre, and J. P. Likforman, "Amplitude and phase measurements of femtosecond pulses shaped by use of spectral hole burning in free-base naphthalocyaninedoped films," J. Opt. Soc. Am. B 20, 1555-1558 (2003).

10. A. M. Weiner, D. E. Leaird, D. H. Reitze, and E. G. Paek, "Femtosecond spectral holography," IEEE J. Quantum Electron. 28, 2251-2261 (1992).

11. D. M. Marom, D. Panasenko, R. Rokitski, P.-C. Sun, and Y. Fainman, "Time reversal of ultrafast waveforms by wave mixing of spectrally decomposed waves," Opt. Lett. 25, 132-134 (2000).

12. M. Tsang and D. Psaltis, "Spectral phase conjugation by quasi-phase-matched three-wave mixing," Opt. Commun. 242, 659-664 (2004).

13. M. Tsang and D. Psaltis, "Spectral phase conjugation with cross-phase modulation compensation," Opt. Express 12, 2207-2219 (2004).

14. M. Tsang and D. Psaltis, "Spontaneous spectral phase conjugation for coincident frequency entanglement," Phys. Rev. A 71, 043806 (2005).

15. Z. D. Walton, M. C. Booth, A. V. Sergienko, B. E. A. Saleh, and M. C. Teich, "Controllable frequency entanglement via auto-phase-matched spontaneous parametric downconversion,” Phys. Rev. A 67, 053810 (2003).

16. V. Giovannetti, L. Maccone, J. H. Shapiro, and F. N. C. Wong, "Generating entangled two-photon states with coincident frequencies," Phys. Rev. Lett. 88, 183602 (2002).

17. O. Kuzucu, M. Fiorentino, M. A. Albota, F. N. C. Wong, and F. X. Kaertner, "Two-photon coincident frequency entanglement via extended phase matching," Phys. Rev. Lett. 94, 083601 (2005).

18. F. Konig and F. N. C. Wong, "Extended phase matching of second-harmonic generation in periodically poled $\mathrm{KTiOPO}_{4}$ with zero group-velocity mismatch," Appl. Phys. Lett. 84, 1644-1646 (2004)

19. R. Kompfner and N. T. Williams, "Backward-wave tubes," Proc. IRE 41, 1602-1611 (1953).

20. H. Heffner, "Analysis of the backward-wave traveling-wave tube," Proc. IRE 42, 930-937 (1954).

21. N. M. Kroll, "Excitation of hypersonic vibrations by means of photoelastic coupling of high-intensity light waves to elastic waves," J. Appl. Phys. 36, 34-43 (1965).

22. D. Bobroff, "Coupled-mode analysis of phonon-photon parametric backward-wave oscillator," J. Appl. Phys. 36, 1760-1769 (1965).

23. S. E. Harris, "Proposed backward wave oscillation in the infrared," Appl. Phys. Lett. 9, 114-116 (1966).

24. Y. J. Ding, S. J. Lee, and J. B. Khurgin, "Transversely pumped counterpropagating optical parametric oscillation and amplification," Phys. Rev. Lett. 75, 429-432 (1995).

25. A. Yariv, Quantum Electronics (Wiley, 1989).

26. V. Giovannetti, S. Lloyd, and L. Maccone, "Quantumenhanced positioning and clock synchronization," Nature 412, 417-419 (2001).

27. G. A. Durkin, C. Simon, and D. Bouwmeester, "Multiphoton entanglement concentration and quantum cryptography," Phys. Rev. Lett. 88, 187902 (2002). 
28. R. A. Fisher, B. R. Suydam, and B. J. Feldman, "Transient analysis of Kerr-like phase conjugators using frequencydomain techniques," Phys. Rev. A 23, 3071-3083 (1981).

29. B. van der Pol and H. Bremmer, Operational Calculus Based on the Two-sided Laplace Integral (Cambridge U. Press, 1964).

30. D. M. Pepper and R. L. Abrams, "Narrow optical bandpass filter via nearly degenerate four-wave mixing," Opt. Lett. 3, 212-214 (1978).

31. N. E. Yu, J. H. Ro, M. Cha, S. Kurimura, and T. Taira, "Broadband quasi-phase-matched second-harmonic generation in $\mathrm{MgO}$-doped periodically poled $\mathrm{LiNbO}_{3}$ at the communications band," Opt. Lett. 27, 1046-1048 (2002).

32. H.-Y. Fan and N.-Q. Jiang, "Special two-mode unitary transform and maximum entanglement state for four wave mixing," Phys. Scr. 71, 277-279 (2005).

33. A. Lamas-Linares, J. C. Howell, and D. Bouwmeester, "Stimulated emission of polarization-entangled photons," Nature 412, 887-890 (2001)

34. G. Michaeli and A. Arie, "Optimization of quasi-phasematched non-linear frequency conversion for diffusion bonding applications," Appl. Phys. B 77, 497-503 (2003).

35. G. P. Agrawal, Nonlinear Fiber Optics (Academic, 2001). 